\title{
Heterochronous Metastases of Lung Adenocarcinoma to Pancreas and Liver: A Case Report from Pathological Perspectives
}

\section{Bo Zhang (iD) ${ }^{\text {,* }}$ \\ Qida $\mathrm{Hu}$ (DD $^{2, *}$ \\ Jiajie $\mathrm{Yu}(\mathrm{DD})$ \\ Junsen Wang (iD ${ }^{3}$ \\ Hanjin Yang iD ${ }^{4}$ \\ Jiongbo Lou (iD ${ }^{5}$ \\ Guoying $\mathrm{Cai}$ (iD) ${ }^{6}$ \\ Haifeng Huang (iD) \\ Mengqiu $\mathrm{Xu}$ (iD ${ }^{5}$ \\ Zhaoying Xiao (iD ${ }^{5}$ \\ Yun Zhang (iD) ${ }^{1,2}$}

'Department of Surgery, Shengzhou People's Hospital, Shaoxing, 312400, People's Republic of China; ${ }^{2}$ Department of Hepatobiliary and

Pancreatic Surgery, First Affliated Hospital,

Zhejiang University School of Medicine, Hangzhou, 310003 , People's Republic of China; ${ }^{3}$ Department of Pathology, Shengzhou People's Hospital, Shaoxing, 3/2400, People's Republic of China; ${ }^{4}$ Department of Pathology, First Affiliated Hospital, Zhejiang University School of Medicine, Hangzhou, 310003 , People's Republic of China; ${ }^{5}$ Department of Infectious Diseases, Shengzhou People's Hospital, Shaoxing, 312400, People's Republic of China; ' ${ }^{6}$ Department of Oncology, Shengzhou People's Hospital, Shaoxing, 312400, People's Republic of China

*These authors contributed equally to this work

Correspondence: Yun Zhang

Department of General Surgery,

Shengzhou People's Hospital, 666 Dangui

Road, Shaoxing, 312400, People's

Republic of China

Tel +8657583338570

Email bigzyun@hotmail.com

Zhaoying Xiao

Department of Infectious Diseases,

Shengzhou People's Hospital, 666 Dangui

Road, Shaoxing, 3/2400, People's

Republic of China

Tel +8657583338276

Email xiaozy1998@।26.com

\begin{abstract}
Immunohistochemistry (IHC) is a vital tool to distinguish tumor metastases from primary lesions in addition to morphologic analysis. In this study, a 64-year-old female with a past surgical history of lung adenocarcinoma 11 years ago was presented with recurrence of liver nodular lesions after multiple surgical procedures, including the Whipple procedure for pancreatic head adenocarcinoma and cytoreductive surgery for liver metastasis. Liver biopsy and review of the previous specimens, based on IHC analyses, suggested heterochronous metastases of lung adenocarcinoma to the digestive systems in a long-time span, instead of primary pancreatic adenocarcinoma. This case demonstrates the potential for misdiagnoses from morphologic analysis alone and suggests the necessity of IHC analyses to avoid misjudgment on tumor phenotypes, when a previous oncologic history is presented.
\end{abstract}

Keywords: heterochronous metastases, lung adenocarcinoma, immunohistochemistry

\section{Introduction}

Pathology is the golden standard of diagnostic works in routine clinical settings. Morphologic pathology is fundamental for current diagnostic studies. In most circumstances, the morphologic criteria for cancer diagnosis do not encompass cancer's proposed biologic hallmarks. ${ }^{1}$ Immunohistochemistry (IHC) assay, which visualizes explicitly the distribution and the amount of particular molecular markers in the tissue using specific antigen-antibody reaction, is a valuable adjunct for morphologic diagnosis. ${ }^{2}$ IHC is widely applied when the initial morphologic diagnosis is unclear, helping pathologists determine the final diagnosis based on molecular profiling.

IHC is also a vital tool to distinguish tumor metastases from primary lesions. For metastatic carcinoma of unknown primary origin, the pathologic approach is stepwise and considers information from its clinical context, morphology, and IHC if necessary. ${ }^{3}$ New emerging characteristic markers combined with established strategies in IHC could narrow down the range of suspected primary malignancies, allowing rapid and accurate identification of the primary origin. ${ }^{4}$ Sometimes without the IHC technology, it is hard to distinguish heterochronic metastases from primary tumors when a landscape review of past oncology history is absent.

In this study, we report a case demonstrating heterochronic metastases of lung adenocarcinoma to the digestive systems over ten years, where morphologic study alone without IHC assay failed to establish a correct diagnosis. 


\section{Case Presentation}

A 64-year-old Asian female was presented to our hospital with a recurrence of liver nodular lesions after multiple surgical resections.

The patient had received lung lobectomy due to lung cancer in the right upper lobe 11 years ago (Figure 1A). Postoperative pathology showed a $4.0 \times 3.9 \times 3.0 \mathrm{~cm}$ moderately differentiated adenocarcinoma, with one positive mediastinal lymph node among all the 10 dissected lymph nodes (Figure 1B). She quit adjuvant chemotherapy after one cycle due to intolerance. No elevation of tumor markers had been reported perioperatively.

Seven years ago, the patient developed jaundice. CT assessment showed a pancreatic head mass with involvement of the distal common bile duct (Figure 2A). Results of tumor markers including CA19-9 were negative. She was then treated with radical pancreaticoduodenectomy, where a $2.5 \times 2.0 \times 2.0 \mathrm{~cm}$ moderately differentiated adenocarcinoma with negative lymph node metastasis was removed (Figure 2B). Afterward, she received adjuvant S-1 chemotherapy (consisting of tegafur $40 \mathrm{mg}$, gimeracil $11.6 \mathrm{mg}$, and oteracil $39.2 \mathrm{mg}$, administered orally twice a day) for 3 cycles ( 2 weeks per cycle followed by a oneweek rest period), which was also discontinued due to adverse events.

The patient was then put on surveillance with an annual imaging assessment. Four years after the Whipple procedure, a follow-up examination showed a solitary liver lesion in segment VII (Figure 3A). She refused interventional treatment or chemotherapy and chose cytoreductive surgery to resect the segment VII lesion. Postoperative pathology demonstrated a $3.0 \mathrm{~cm}$ segment VII adenocarcinoma and suggested the diagnosis of liver metastasis (Figure 3B).

Recently, the patient was admitted for the recurrence of a liver lesion, along with multiple lung nodules (Figure 4A). Recurrence of the previous adenocarcinoma in the liver and lungs was the first impression. The tumor board reviewed her past medical history and proposed the possibility of metastases of the lung adenocarcinoma to the pancreas, liver, and lungs. Liver biopsy confirmed adenocarcinoma, with similar morphology, such as the acinar and tubular structures, to the previous specimens (Figure 4B). Further immunohistochemical studies demonstrated that the lung adenocarcinoma eleven years ago, the pancreatic adenocarcinoma seven years ago, the liver adenocarcinoma three years ago, and current liver adenocarcinoma all shared features of positive thyroid transcription factor-1 (TTF-1, clone SPT24) and Napsin
A expressions (Figure 4C and D), indicating the exact pathological origin of the lung adenocarcinoma.

We then tested the adenocarcinoma specimen for mutations of driver genes in lung cancers as well as in the metastatic liver adenocarcinoma, and found an epidermal growth factor receptor (EGFR) exon 19 non-frameshift deletion, which was associated with favorable prognosis, ${ }^{5}$ in both samples. The patient was then administrated with gefitinib, a tyrosine kinase inhibitor for lung cancers with EGFR exon 19 mutations. ${ }^{6}$ Imaging reassessment one month later indicated a status of partial response, with the lung nodules disappeared and the liver metastasis significantly shrunk.

\section{Discussions}

Our case report described a rare situation - heterochronous metastases of lung adenocarcinoma to pancreas and liver over a long time span. The initial misdiagnosis was caused by missing detailed descriptions of the past medical history and not conducting IHC assays, as the record only showed "adenocarcinoma" without specific identification of its phenotype or tumor origin. Besides, morphologic pathology alone could not differentiate a metastatic tumor from a primary lesion. This case reminds us to remain vigilant about possible heterochronous metastases, especially in patients with a previous tumor history.

Lung adenocarcinoma is the most commonly diagnosed histological subtype of non-small-cell lung cancer, a predominant cause of cancer-related mortality. ${ }^{7}$ Extrathoracic metastases are widely found in the bones, brain, liver, and adrenal glands. ${ }^{8,9}$ Pancreatic metastases from lung adenocarcinoma have also been reported before, ${ }^{10-15}$ but the pancreatic lesions in most cases were presented as synchronous metastases. Heterochronous metastases in the pancreas, which were relatively rare, might be easily confused with primary pancreatic malignancy since adenocarcinoma is also a mainstream phenotype of pancreatic carcinoma.

The critical flaw in the current diagnostic approach is the absence of IHC assays for the pancreatic tumor from the Whipple procedure. In addition to the morphological pathology, IHC assays should be routinely performed for patients with previous oncologic history and tumors without clear origin. ${ }^{16}$ The secondary flaw is the omission of pathological comparison in morphology between the pancreatic tumor and the primary lung adenocarcinoma. The comparison of the current and past tumor pathological results should be established to be a standard operating 

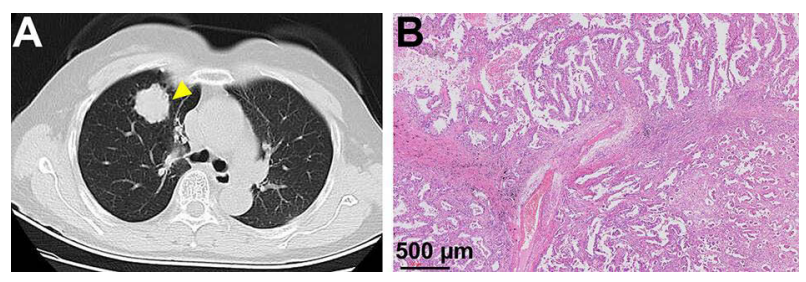

Figure I (A) CT image and (B) postoperative pathology of lung adenocarcinoma II years ago. The yellow triangle indicates the location of lung adenocarcinoma.
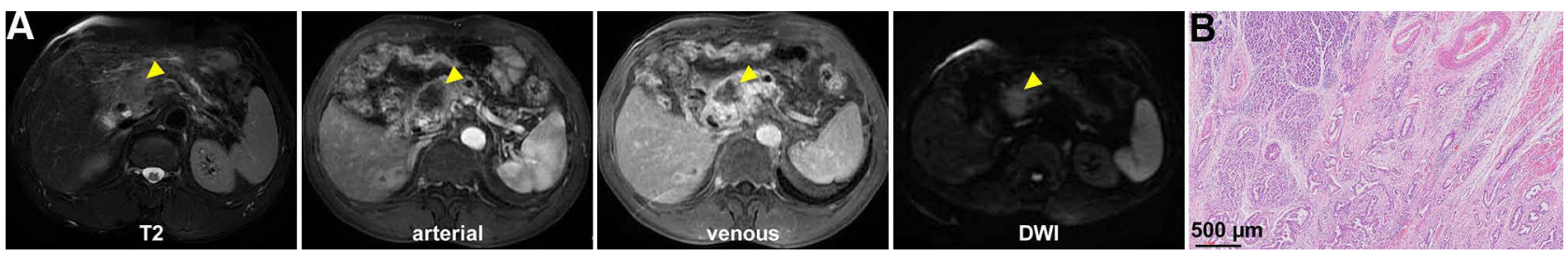

Figure 2 (A) MRI investigation with T2-weighted, arterial phase, venous phase images, and diffusion-weighted imaging (DWI), and (B) postoperative pathology of pancreatic mass 7 years ago. The yellow triangles indicate the location of pancreatic mass.

procedure for patients with previous oncologic problems to reduce similar mistakes.

The long-term survival after the pancreaticoduodenectomy is also a hint for the pathologists that some primary pancreatic cancers might be misdiagnosed. Pancreatic carcinoma features a poor prognosis with a 5-year survival of approximately $10 \% .{ }^{17,18}$ On the contrary, localized lung adenocarcinoma, if treated promptly at an early stage, has a much better survival profile with a 5-year survival rate of around $50 \%{ }^{19,20}$ In our case, EGFR exon 19 deletion, a mutation subtype associated with longer progressionfree survival, ${ }^{21}$ was found, which might also contribute to the late progression.

Meanwhile, the principle of monism also applied to the identification of malignancy origin in this case. According to Occam's razor, the diagnosis of lung adenocarcinoma with heterochronous pancreatic and liver metastases should have a higher possibility to be true other than the complex situation of multiple primary tumors.

In conclusion, we reported an educational case where heterochronous pancreatic and liver metastases from a previous lung adenocarcinoma were misdiagnosed as primary pancreatic adenocarcinoma based on morphological analyses alone without IHC assays. This case suggests the necessity of IHC analyses for both current and archived pathology samples to avoid misjudgment on tumor phenotypes when a previous oncologic history is presented.

\section{Ethics Approval and Consent for Publication}

This study was approved by the research ethics committee of Shengzhou People's Hospital, and written informed
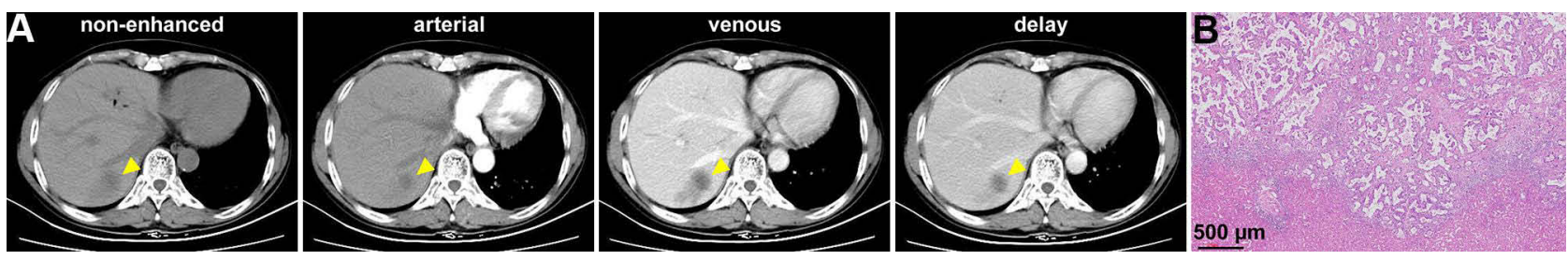

Figure 3 (A) CT investigation with non-enhanced, arterial phase, venous phase, and delayed phase images, and (B) postoperative pathology of liver mass 3 years ago. The yellow triangles indicate the location of liver mass. 


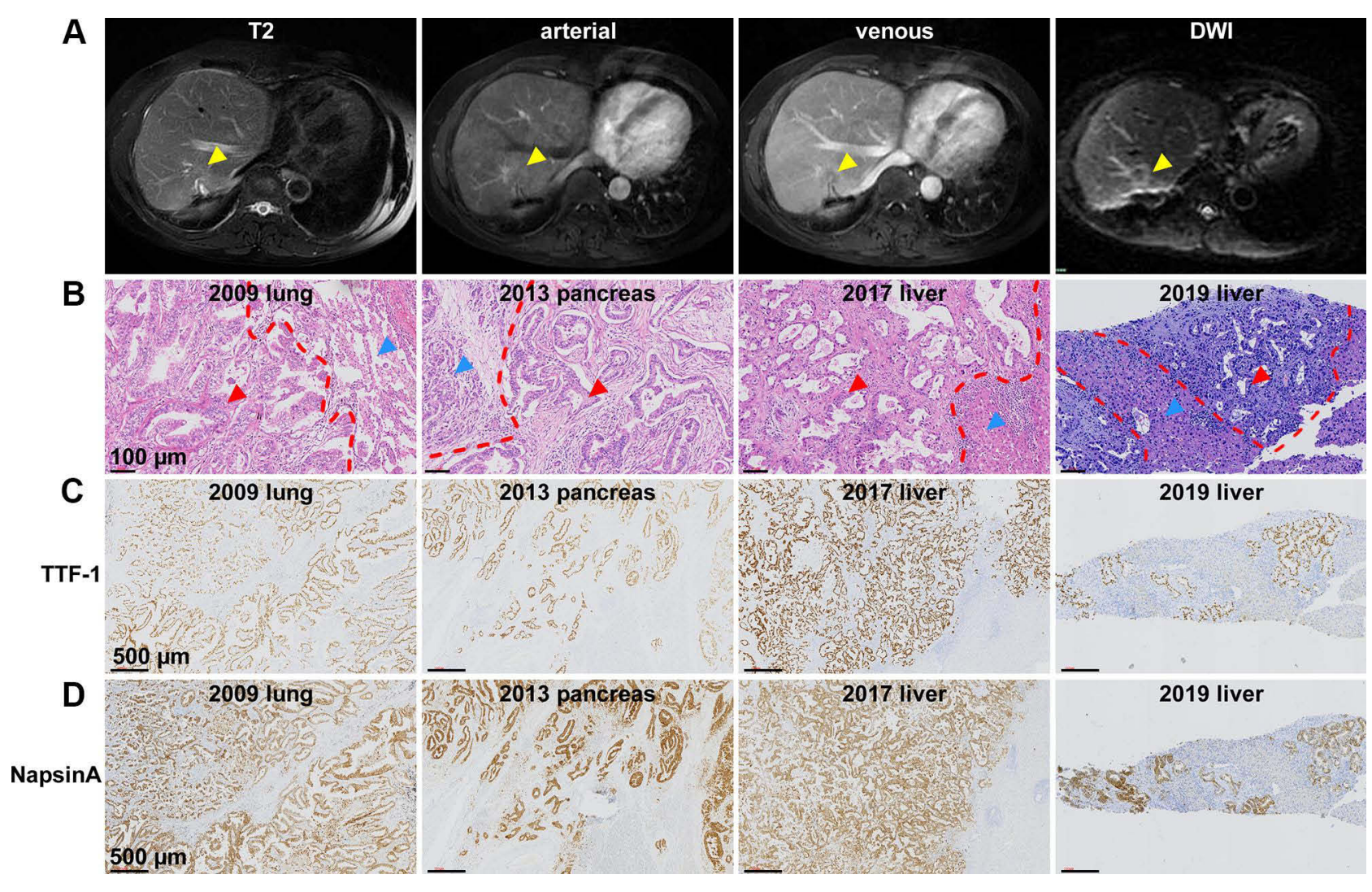

Figure 4 (A) MRI investigation with T2-weighted, arterial phase, venous phase, and DWI images. The yellow triangles indicate the location of recurrent liver mass. (B) Similar morphology in the four heterochronous specimen, namely the lung adenocarcinoma II years ago, the pancreatic mass 7 years ago, the liver mass 3 years ago, and the recurrent liver mass. The red triangles indicate the acinar and tubular structures in the adenocarcinoma, and the blue triangles indicate the original tissue structures. The bars indicate $100 \mu \mathrm{m}$. (C) IHC studies on TTF-I and (D) NapsinA expressions were performed in the four heterochronous specimen. The bars indicate $500 \mu \mathrm{m}$.

consent for publication of the clinical details and images was obtained from the patient.

\section{Informed Consent Statement}

Informed written consent was obtained from the patient for publication of this report and any accompanying images.

\section{Acknowledgment}

We thank the Shengzhou hepatobiliary and pancreatic multidisciplinary board for the assistance and helpful advice. We also appreciate language polishing by Ivy $\mathrm{Wu}$ from Harvard T.H. Chan School of Public Health.

\section{Author Contributions}

Zhang $\mathrm{B}$ and $\mathrm{Hu} \mathrm{Q}$ treated the patient, reviewed the related literature, drafted the manuscript, and contributed equally to this work; Yu J, Lou J, Cai G, Huang $\mathrm{H}$, and $\mathrm{Xu} \mathrm{M}$ participated in clinical treatment and collected the data; Wang $\mathrm{J}$ and Yang $\mathrm{H}$ performed the pathological analyses and interpretation; Zhang Y, Xiao $\mathrm{Z}$, and $\mathrm{Hu} \mathrm{Q}$ were responsible for the revision of the manuscript for important intellectual content. All authors made a significant contribution to the work reported, whether that is in the conception, study design, execution, acquisition of data, analysis and interpretation, or in all these areas; took part in drafting, revising or critically reviewing the article; gave final approval of the version to be published; have agreed on the journal to which the article has been submitted; and agree to be accountable for all aspects of the work.

\section{Funding}

This work was financially supported by the National Natural Science Foundation of China (No. 81972207), Medical Health Science and Technology Project of Zhejiang Provincial Health Commission (2021453721), and the Shengzhou Technology Program (No. 201909). 


\section{Disclosure}

No potential conflict of interest was reported by the authors.

\section{References}

1. Idikio HA. Immunohistochemistry in diagnostic surgical pathology: contributions of protein life-cycle, use of evidence-based methods and data normalization on interpretation of immunohistochemical stains. Int J Clin Exp Pathol. 2009;3:169-176.

2. Kim SW, Roh J, Park CS. Immunohistochemistry for pathologists: protocols, pitfalls, and tips. J Pathol Transl Med. 2016;50:411-418. doi:10.4132/jptm.2016.08.08

3. Oien KA. Pathologic evaluation of unknown primary cancer. Semin Oncol. 2009;36:8-37. doi:10.1053/j.seminoncol.2008.10.009

4. Conner JR, Hornick JL. Metastatic carcinoma of unknown primary: diagnostic approach using immunohistochemistry. Adv Anat Pathol. 2015;22:149-167. doi:10.1097/PAP.0000000000000069

5. Devarakonda S, Morgensztern D, Govindan R. Genomic alterations in lung adenocarcinoma. Lancet Oncol. 2015;16:e342-51. doi:10.1016/S1470-2045(15)00077-7

6. Sharma SV, Bell DW, Settleman J, Haber DA. Epidermal growth factor receptor mutations in lung cancer. Nat Rev Cancer. 2007;7:169-181. doi:10.1038/nrc2088

7. Sung H, Ferlay J, Siegel RL, et al. Global cancer statistics 2020: GLOBOCAN estimates of incidence and mortality worldwide for 36 cancers in 185 countries. CA Cancer J Clin. 2021;71(3):209-249. doi: $10.3322 /$ caac. 21660

8. Wang X, Wang Z, Pan J, et al. Patterns of extrathoracic metastases in different histological types of lung cancer. Front Oncol. 2020;10:715. doi: 10.3389 /fonc. 2020.00715

9. Stenbygaard LE, Sorensen JB, Olsen JE. Metastatic pattern at autopsy in non-resectable adenocarcinoma of the lung: a study from a cohort of 259 consecutive patients treated with chemotherapy. Acta Oncol. 1997;36:301-306. doi:10.3109/02841869709001267

10. Kageyama Y, Yamaguchi R, Watanabe S, et al. A long-term survival case after resection of the pancreatic metastasis from lung cancer. Int J Surg Case Rep. 2019;61:222-225. doi:10.1016/j. ijscr.2019.07.060
11. Wang W, Kan Y, Yang X, Liu J, Yang J. Concurrent pancreatic metastasis from lung adenocarcinoma and primary cholangiocarcinoma on FDG PET/CT imaging. Clin Nucl Med. 2019;44 (5):426-428. doi:10.1097/RLU.0000000000002496

12. Hayasaka K, Shiono S, Yanagawa N, Yarimizu K, Suzuki K, Endoh M. Hemorrhage of a pancreatic metastasis from lung adenocarcinoma after osimertinib therapy. J Thorac Dis. 2018;10(9):E686E689. doi:10.21037/jtd.2018.08.108

13. Furuya T, Shimada J, Okada S, Tsunezuka H, Kato D, Inoue $M$ Successful treatment with afatinib for pancreatic metastasis of lung adenocarcinoma: a case report. J Thorac Dis. 2017;9(10):E890 E893. doi:10.21037/jtd.2017.09.123

14. Mori N, Sawada T, Satoh H, Kawaguchi M, Hara H, Matsushita K. A resected case of solitary pancreatic metastasis from adenocarcinoma of the lung. JOP. 2008;9:698-703.

15. Igai H, Kamiyoshihara $M$, Nagashima T, Ohtaki $Y$, Shimizu K. A resectable pancreatic metastasis from pulmonary adenocarcinoma. Ann Thorac Cardiovasc Surg. 2014;20(3):243-245. doi:10.5761/atcs. cr.12.02049

16. Selves J, Long-Mira E, Mathieu MC, Rochaix P, Ilie M. Immunohistochemistry for diagnosis of metastatic carcinomas of unknown primary site. Cancers. 2018;10(4):108. doi:10.3390/ cancers 10040108

17. Wolfgang CL, Herman JM, Laheru DA, et al. Recent progress in pancreatic cancer. CA Cancer J Clin. 2013;63(5):318-348. doi: $10.3322 /$ caac. 21190

18. Mizrahi JD, Surana R, Valle JW, Shroff RT. Pancreatic cancer. Lancet. 2020;395:2008-2020. doi:10.1016/S0140-6736(20)30974-0

19. Lu T, Yang X, Huang Y, et al. Trends in the incidence, treatment, and survival of patients with lung cancer in the last four decades. Cancer Manag Res. 2019;11:943-953. doi:10.2147/CMAR.S187317

20. Lu C, Bera K, Wang X, et al. A prognostic model for overall survival of patients with early-stage non-small cell lung cancer: a multicentre, retrospective study. Lancet Digit Health. 2020;2:e594-e606. doi:10.1016/s2589-7500(20)30225-9

21. Sutiman N, Tan SW, Tan EH, et al. EGFR mutation subtypes influence survival outcomes following first-line gefitinib therapy in advanced asian NSCLC patients. $J$ Thorac Oncol. 2017;12:529-538. doi:10.1016/j.jtho.2016.11.2225
OncoTargets and Therapy

\section{Publish your work in this journal}

OncoTargets and Therapy is an international, peer-reviewed, open access journal focusing on the pathological basis of all cancers, potential targets for therapy and treatment protocols employed to improve the management of cancer patients. The journal also focuses on the impact of management programs and new therapeutic agents and protocols on patient perspectives such as quality of life, adherence and satisfaction. The manuscript management system is completely online and includes a very quick and fair peer-review system, which is all easy to use. Visit http://www.dovepress.com/ testimonials.php to read real quotes from published authors 